\title{
82. Further Study on the Diffraction of Cathode Rays by Mica.
}

\author{
By Seishi KIKUCHI. \\ Institute of Physical and Chemical Research, Tokyo. \\ (Rec. June 9, 1928. Comm. by T. Terada, m.I A., June 12, 1928.)
}

Patterns of the second type: As already mentioned in the preceding note, the second type of pattern is obtained when the thickness of the mica plate is increased. Of course, the transition from the first type to the second takes place continuously with the thickness of the plate and a pattern, which has an intermediate structure, can be obtained. Therefore this classification is only for the sake of convenience of description. In the previous case, the plates used were so thin, that they produced no colour of interference, while in the present case the plates were thick enough to produce bright colour. The structure of the pattern of the second type is much affected by a small change in the orientation of the crystal with respect to the incident beam, while that of the first type remains almost unchanged. This is one of the most remarkable differences in the characteristics of both types.

A pattern of this type generally consists of four elements, viz. spots, rings., black and white lines. In Fig. 1 one of the patterns is shown in which all the four elements are present. As it is difficult to show the details in the reproduced photogram, it is represented schematically in Fig. 2. There are two concentric rings (something like those obtained by G. P. Thomson), the common centre of which is slightly displaced from the central spot. On these rings and also near the central spot, a number of spots are visible. Besides these, there are present a number of black and white lines, which are crossing the pattern in a characteristic manner, the angle between any two lines being mostly an integral multiple of $30^{\circ}$. The distribution of these elements in the pattern changes in some systematic manner as the specimen is gradually tilted, while its dependence upon the applied potential is not so remarkable. For a closer study of these relations, however, the present apparatus is not adequate but an improved one, which is provided with a device to read the orientation of the crystal more accurately is being built. Although, thus far the experiment is only qualitative, the results obtained seems to be in favour of the following view. 

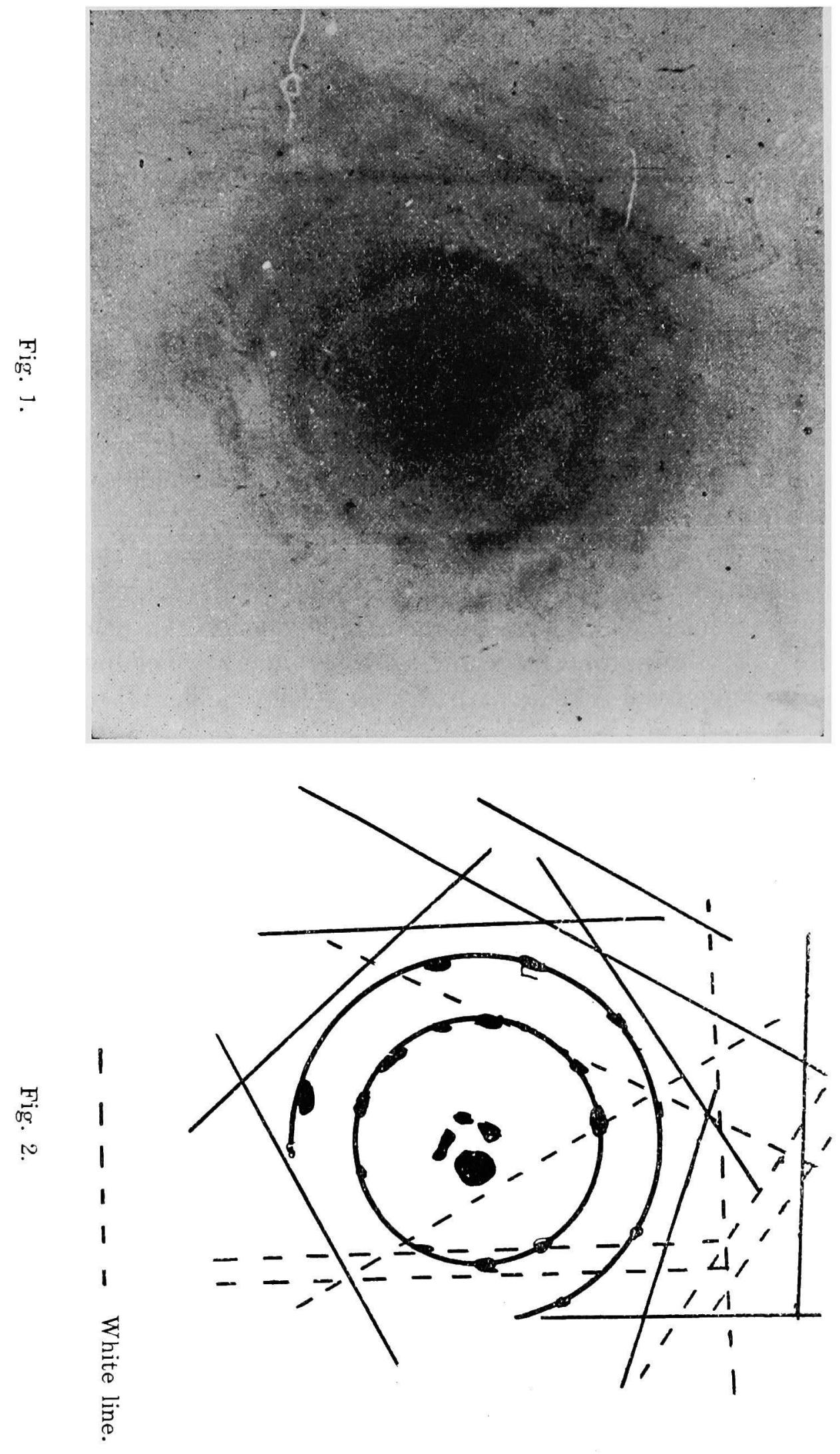
1) There can be little doubt that the spots are similar to the Laue spots, i.e. the interference maxima of space lattice.

2) The configuration of the concentric rings depends on the orientation of the specimen. When the specimen is tilted a little the centre of the rings is displaced from the central spot and their diameters increase. Moreover, another small ring which passes through the central spot and which is concentric to the outer ones becomes noticeable. All these phenomena may be explained, if we assume a diffraction due to an array of atoms along a straight line making a small angle $\alpha$

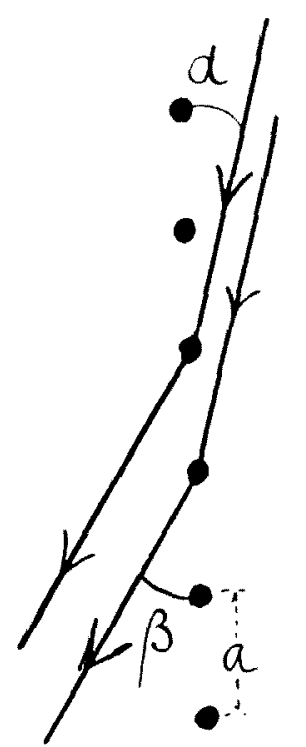

Fig. 3. with the incident beam. If $a$ is the distance between the successive atoms and $\beta$ the angle between a diffracted beam and the array (Fig. 3), then the condition of interference maxima is given by

$$
\cos \beta=\cos \alpha-\frac{n \lambda}{a}
$$

where, $\lambda$ is the wave length of the rays and $n$ an integer. When $n=0$, we have $\alpha=\beta$. Thus the interference fringe of zero order appears as a ring passing through the central spot. On the other hand, when $\alpha=0$,

$$
\sin \frac{\beta}{2}=\sqrt{\frac{n \lambda}{2 a}}
$$

In this case the fringe becomes a series of concentric circles, with their common centre at the central spot. This is nearly the case in Fig. 1. Then by (2), the ratio of the diameters of two rings corresponding to the first and second orders must be $1: \sqrt{2}$. This is proved to be the case for two concentric rings in Fig. 1. The diameter of a ring is proportional to the square root of wave-length, or inversely proportional to the fourth root of the applied potential. This also seems to be supported by the experiment. Moreover, the value of $a$ calculated from the wave-length and the diameters of the rings is just of the magnitude to be expected. The appearance of the Laue spots near the rings may easily be accounted for as in the case of the pattern of the first type. It seems worth noticing that in this case one- and three-dimensional effects of a group of diffracting atoms play separate parts, while in the case of X-rays the latter effect alone is pronounced. It may be connected with some fundamental difference between material and light waves.

3) As regards the black and white lines, it is difficult for the present to give any convincing explanation for their origin. They 
show, however, some resemblance with the reflection and absorption lines obtained by Rutherford and Andrade ${ }^{1)}$ in their experiment on the transmission of a divergent pencil of X-rays through a crystal plate. Thus, they might be explained as the lines of a similar origin, if we take into consideration a multiple scattering of cathode rays in the crystal.

This investigation was carried out at the suggestion of Prof. S. Nishikawa, to whom the author wishes to express his sincerest thanks for his continued interest and valuable criticisms.

1) Rutherford a. Audrade: Phil. Mag., 28 (1914), 263. 